\title{
A genotyping study of human immunodeficiency virus type-1 drug resistance in a small Brazilian municipality
}

\author{
Walter A Eyer-Silva/*/**/+ , Mariza G Morgado
}

\begin{abstract}
Laboratório de Aids \& Imunologia Molecular, Instituto Oswaldo Cruz-Fiocruz, Av. Brasil 4365, 21045-900 Rio de Janeiro, RJ, Brasil *Hospital Universitário Gaffrée e Guinle, Universidade Federal do Estado do Rio de Janeiro, Rio de Janeiro, RJ, Brasil **Programa Municipal de HIV/Aids de Miracema, Miracema, RJ, Brasil
\end{abstract}

\begin{abstract}
In Brazil, surveillance studies on antiretroviral drug resistance among drug-nä̈ve and treatment-experienced patients have focused primarily on patients living in large urban centers. As the epidemic spreads towards small municipalities and the innermost parts of the country, it will be essential to monitor the prevalence of antiretroviral drug resistance in these areas. We report the first survey on the prevalence of antiretroviral drug resistance in a small Brazilian municipality. Between July 1999 and March 2005, 72 adult human immunodeficiency virus type-1(HIV-1)-infected patients received care at the Municipal HIV/AIDS Program of the small, southeastern municipality of Miracema, state of Rio de Janeiro. A genotyping study of antiretroviral drug resistance was performed in 54 patients. Among 27 samples from treatment-experienced patients, $9(33.3 \%)$ harbored strains with reduced drug susceptibility. Among these, 6 had reduced susceptibility to reverse transcriptase (RT) inhibitors and 3 to both $R T$ and protease inhibitors. No primary antiretroviral drug resistance was recorded among 27 drug-naïve subjects. The relatively low prevalence of resistance mutations in the Miracema cohort argues against the concern that resource-poor settings should not implement widespread accessibility to standard of care antiretroviral combinations due to the possibility of sub-optimal adherence leading to the emergence and spread of drug-resistant strains.
\end{abstract}

Key words: antiretroviral resistance - genotyping - human immunodeficiency virus type-1 - small municipalities - subtypes Brazil

Continued human immunodeficiency virus type 1 (HIV1) replication in the presence of selective pressure of drugs targeting the reverse transcriptase (RT) and protease (PR) viral enzymes leads to the emergence of specific point mutations in the RT and PR genomic regions of the polymerase ( $p o l)$ gene and poses one of the major obstacles to the long-term efficacy of antiretroviral therapy. Since strains harboring resistance-associated mutations to a single or to multiple antiretroviral agents can be transmitted both horizontally (Hecht et al. 1998) and vertically (Siegrist et al. 1994), it is essential to monitor the prevalence of resistant strains in the community.

In Brazil, surveillance studies on the prevalence of mutations conferring antiretroviral drug resistance are being reported both from national (Brindeiro et al. 1999, 2003, Tanuri et al. 2002) and regional (Dumans et al. 2002, Pires et al. 2004, Couto-Fernandez et al. 2005, Rodrigues et al. 2005) studies based on large urban centers. However, as the Brazilian epidemic spreads from the large urban centers towards small municipalities and the innermost parts of the country (Szwarcwald et al. 2000), appropriate studies on the features of HIV-1 infection in relatively small Brazilian communities are urgently needed. We report the results of the first survey on antiretroviral drug resistance in a small Brazilian municipality: the southeastern Miracema, in the state of Rio de Janeiro. Since Miracema is located in northwestern Rio de Janeiro, a re-

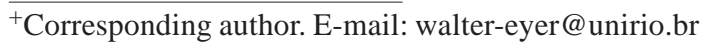
Received 31 May 2005

Accepted 23 November 2005 gion where municipalities are known to have low human development index values (IPEA 2003), the present study is an opportunity to analyze the prevalence of HIV-1 drug resistance in a resource-poor setting with widespread availability of standard of care highly active antiretroviral therapy (HAART) regimens.

\section{MATERIAL AND METHODS}

Patients and setting - Miracema is a small municipality in northwestern state of Rio de Janeiro $\left(21^{\circ} 24^{\prime} 50^{\prime \prime} \mathrm{S}\right.$; $\left.42^{\circ} 11^{\prime} 52^{\prime \prime} \mathrm{W}\right), 280 \mathrm{~km}$ far from the city of Rio de Janeiro, at the border of the state of Minas Gerais. A detailed clinical and epidemiological characterization of the cohort is presented elsewhere (Eyer-Silva et al. 2005). Monthly medical appointments are offered at a local ambulatory facility with a physician based in the city of Rio de Janeiro. Antiretroviral agents are freely supplied to patients when clinically indicated, as part of the national AIDS Program of the Ministry of Health. Patients were staged according to the 1993 Revised Classification System of the Centers for Disease Control and Prevention (CDC 1992). The study protocol was reviewed and approved by the Ethics Review Board at Instituto de Pesquisas Clínicas Evandro Chagas, Fundação Oswaldo Cruz, Rio de Janeiro.

Nucleotide sequencing - After obtaining signed informed consent, blood samples were collected from adult patients between December 2001 and March 2005. Total genomic DNA was extracted from $200 \mu \mathrm{l}$ of buffy coat using a QIAmpl Blood Kit (Qiagen Inc., Chatswoth, CA, US), according to manufacturer's instructions. DNA samples $( \pm 1 \mu \mathrm{g})$ were PCR-amplified by a nested protocol in a Perkin Elmer 480 or 9600 Thermal Cycle. A fragment of the HIV-1 pol gene, spanning both PR and RT regions, 
was amplified in the following mixture: $5 \mu 1$ of extracted DNA, $5 \mu \mathrm{l}$ of $10 \times$ Taq buffer, $0.6 \mu \mathrm{l}$ of $25 \mathrm{mM}$ (each) deoxynucleoside triphosphates, $5 \mu \mathrm{l}$ of $\mathrm{MgCl}_{2} 25 \mathrm{mM}, 1 \mu \mathrm{l}(25$ pM) of each PCR-primer, $33 \mu \mathrm{l}$ of $\mathrm{H}_{2} 0$, and $0.5 \mu \mathrm{l}$ of $\mathrm{Taq}$ DNA polymerase (Amersham Pharmacia Biotech Inc.). An initial cycle was performed with a denaturation temperature set at $95^{\circ} \mathrm{C}(3 \mathrm{~min})$, annealing set at $55^{\circ} \mathrm{C}(1 \mathrm{~min})$ and extension set at $72^{\circ} \mathrm{C}(1 \mathrm{~min})$, followed by 35 cycles with denaturation at $95^{\circ} \mathrm{C}(1 \mathrm{~min})$, annealing at $55^{\circ}(45 \mathrm{~s})$ and extension at $72^{\circ} \mathrm{C}(1 \mathrm{~min})$. A final extension of $10 \mathrm{~min}$ was set at $72^{\circ} \mathrm{C}$. For second-round PCR, a $5 \mu$ aliquot of the first-round PCR mixture was used. Oligonucleotides DP10 (5'-TAACTCCCTCTCAGAAGCAGGAGCCG-3') and RT12 (5'-ATCAGGATGGAGTTCATAACCCATCCA-3') were used as sense and antisense outer primers, respectively. Oligonucleotides DP16 (5'-CCTCAAATCACTCTTT GGCAAC-3') and RT4 (5'-AGTTCATAACCCATCC AAAG-3') were used as sense and antisense nested primers, respectively. Amplification results were checked on agarose gel electrophoresis and ethidium bromide staining. The PCR products were then purified with the commercial Wizard SV Gel and PCR Clean-Up System (Promega, Madison, WI, US) and sequenced in an automated ABI 310 or 3100 automated sequencer (Applied Biosystems, Foster City, CA, US) using primers DP16, RT4, LR49 (5'-CAATGG CCATTGACAGAAGA-3') and L51 (5'TGTGGTAT CCTAATTGAACTTCCC-3') for pol fragments. Sequencing reaction mixtures were assembled with BigDye Terminator v.3.0 Cycle Sequencing Reaction Kits (Applied Biosystems). Chromatogram sequencing files were inspected with Chromas 1.45 (Technelysium Ltd., Queensland, Australia) and contigs were assembled by using SeqMan II, included in the DNASTAR software package (Promega) (Burland 2000). Sequences were deposited on the GenBank database under the accession numbers AY929012 to AY929061 and DQ058780 to DQ058783.

Drug resistance genotyping - Previously described drug-resistance associated mutations in the PR and RT genes were sought and genotyping results were interpreted by using the drug resistance interpretation beta test from the HIV RT and PR Sequence Database, Stanford University, version 4.1 (Kantor et al. 2001).

Analysis of sequences and phylogenetic studies - For subtyping analysis, sequences were aligned against a set of reference strains from all known HIV-1 group M subtypes (gathered from the Los Alamos HIV Database: http:/ /hiv-web.lanl.gov) and trimmed to equivalent lengths by using CLUSTAL X (Thompson et al. 1997). A SIVcpz sequence was used as outgroup. An alignment of $845 \mathrm{bp}$ (corresponding to positions 2364 to 3198 relative to HXB2 genome, GenBank accession no. K03455) was obtained. Phylogenetic inferences were performed by the neighborjoining (NJ) algorithm (Saitou \& Nei 1987) based on a DNA distance matrix and using the F84 model of nucleotide substitution (Felsenstein 1984) implemented in PAUP* version 4.0b10 (Swofford 2002). The robustness of the trees was evaluated by bootstrap analysis (Felsenstein 1985) with 100 rounds of replication. The bootscanning method was used to detect and study re- combination, as implemented in the SIMPLOT software, version 2.5 (Salminen et al. 1995). The analysis was performed on a sliding window of 400 nucleotides of the query sequences moving by increments of 20 nucleotides along an alignment of the reference sequences.

\section{RESULTS}

Between July 1999 and January 2005 a total of 72 HIV1 infected adult patients ( 37 female) received care at the Municipal HIV-1/AIDS Program. Out of the 58 patients from whom a blood sample was available, $p o l$ sequences were obtained from 54 (we failed to obtain $\mathrm{pol}$ sequences from 4 samples, even after analysis of a second blood sample). Fifty samples were assigned subtype B, whereas strains M02, M08, M31, and M36 were BF1 mosaic forms (the first 3 sharing common intersubtype breakpoints). These BF1 recombinants will be further described elsewhere. No other subtypes were found.

The Table presents the demographic, epidemiological, clinical and virological data of 54 studied patients. As of sample collection, 27 patients were treatment-naïve, 23 had already been exposed to HAART and 4 were not on therapy but had been previously offered vertical transmission prophylaxis (VTP) with a combination of zidovudine, lamivudine and nevirapine. Among treatment-experienced patients, 10 were on a combination of 2 nucleoside RT inhibitors (NRTI) plus 1 non-nucleoside RT inhibitor (NNRTI), 9 were on a regimen of 2 NRTI plus 1 PR inhibitor (PI), and 4 were on a 2 NRTI plus 1 PI plus 1 NNRTI combination. A total of 29 patients were on an AIDS-defining CDC stage category ( 27 on stage $\mathrm{C}$ and 2 on stage A3).

Among the 27 treatment-exposed patients, 9 (33.3\%) harbored strains with reduced susceptibility to antiretroviral drugs. Among these, 3 had resistance mutations against NRTI and PI agents (patients M05, M11 and M12, all of whom had been exposed to PI-based regimens), 4 had mutations associated with NRTI resistance (patients M07, M14, M20, and M31), and 2 had NNRTI-associated mutations (patients M08 and M25). The most common mutations associated with NRTI were M184V (4 strains), and the thymidine-associated mutations D67N (2 cases), K70R ( 2 cases) and K219/E/Q ( 2 cases). Isolated cases of mutations T69S, M41L and L210W were also recorded. Resistance mutations to NNRTI were found in samples M08 (Y181C and G190A) and M25 (K103N). Mutations conferring reduced susceptibility to PI were found in samples M05, M11 and M12. Patients M05 and M12 had long treatment histories that included sequential use of 4 and 3 consecutive PI agents, respectively. Recorded resistance mutations in the PR gene were A71T and N88S/D (in all 3 cases). Among the 4 female patients who had previously received antiretroviral therapy for vertical transmission prophylaxis, none had reduced drug susceptibility, although sample M33 harbored the polymorphism V106I and the atypical mutation P225L, none of which are currently associated with resistance. Overall, the common polymorphisms M36I, L63P, and V77I were observed in 9 (16.6\%), 34 (70\%) and $13(24 \%)$ samples, respectively. All strains recovered from the 27 treatment-naïve patients were considered susceptible to NRTI, NNRTI, and PI. 


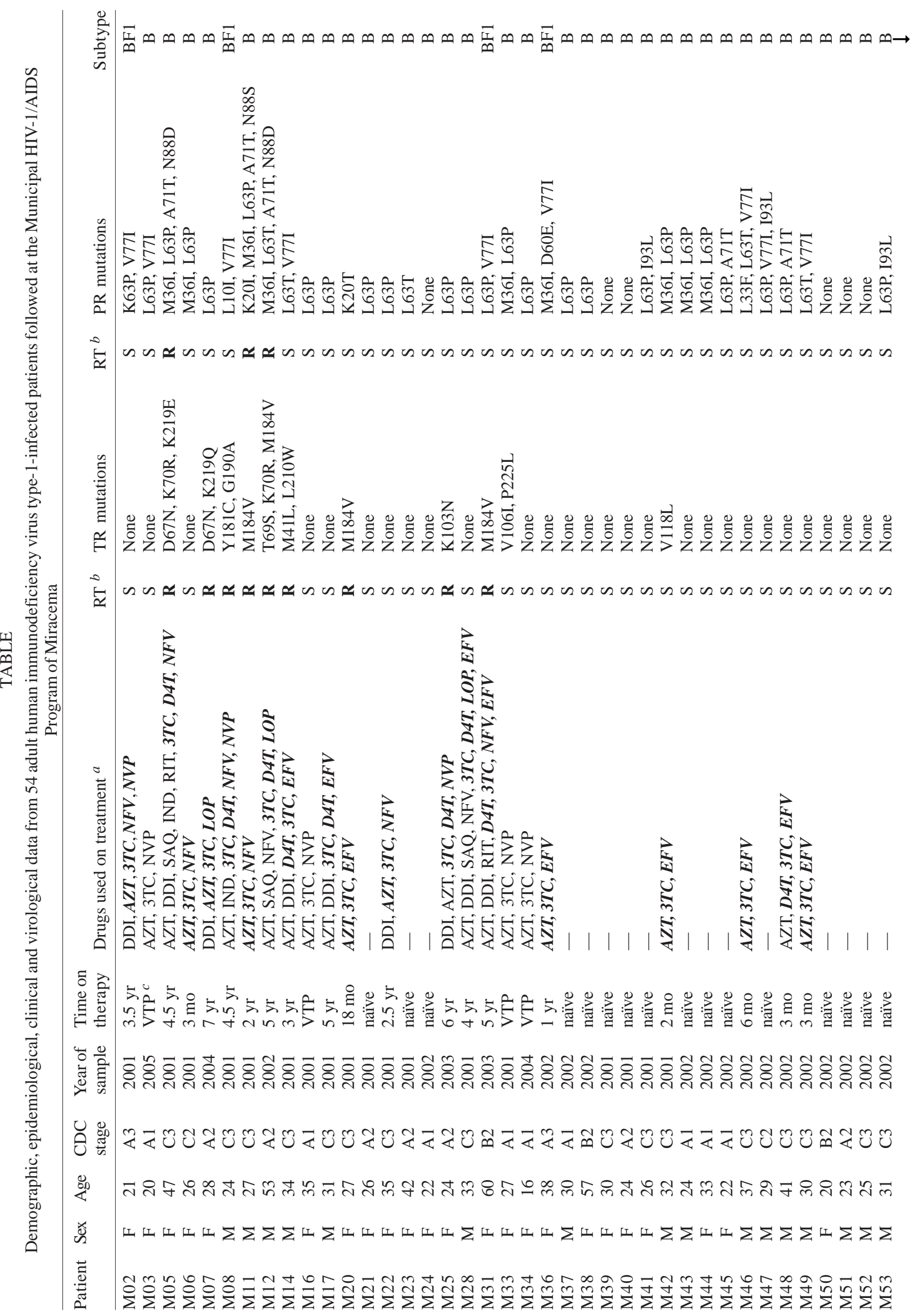




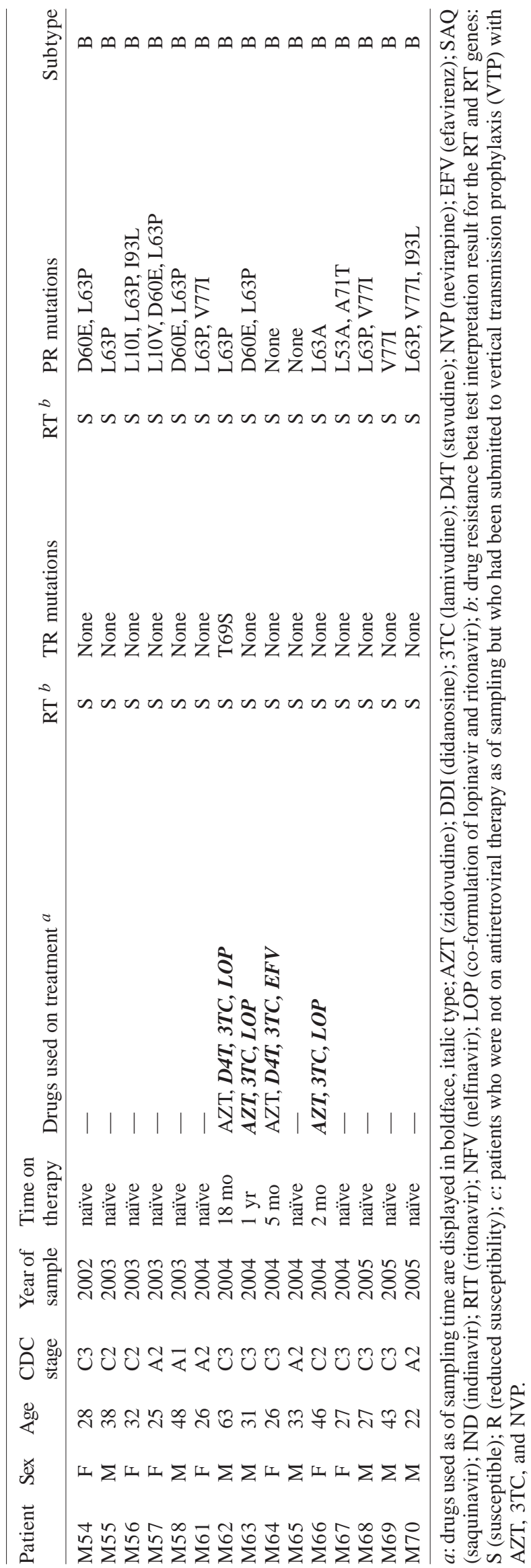

\section{DISCUSSION}

Little is known about the features of HIV-1 infection in small Brazilian municipalities and rural areas. As the epidemic spreads from the large urban centers towards small municipalities and the innermost parts of the country (Szwarcwald et al. 2000, Brito et al. 2001), there is an urgent need to study the clinical, epidemiological and virological characteristics of HIV-1 infection in these settings. These studies will be of utmost importance to optimize the institution of adequate control measures, grasp the true magnitude of the problem, improve clinical recognition and management of HIV-1 infection and better allocate resources.

The present report is the first survey of antiretroviral drug resistance in a small Brazilian municipality. Like all similar studies in the field, it should be interpreted with the understanding that resistance testing is insensitive to minor drug-resistant variants and thus may fail to detect strains resistant to previously used drugs and to detect decayed resistant variants transmitted to untreated subjects.

Brazilian surveillance studies on the prevalence of resistance mutations in treatment-experienced patients have reported variable prevalence figures of reduced susceptibility to antiretroviral agents. Mutation M184V, for instance, associated with high-level resistance to the NRTI lamivudine, has been recently reported in 67.7, 64, and $18 \%$ of patients failing HAART in the states of Rio de Janeiro (Couto-Fernandez et al. 2005), São Paulo (Rodrigues et al. 2005), and the Federal District (Cerqueira et al. 2004), respectively. Prevalence figures of resistance mutations are to be interpreted taking into consideration current local prescribing patterns, as well as recommendations, eligibility and access to HIV resistance testing for patient management.

In the present Miracema cohort, we found an overall low prevalence of reduced susceptibility to antiretroviral agents among 27 treatment-exposed patients. We recorded 9 samples with reduced susceptibility against RT inhibitors and 3 against PI. The prevalence of thymidine-associated mutations (4 cases) and of the M184V mutation (4 cases) clearly reflects the frequency with which combinations that include thymidine analogues (zidovudine and stavudine) and lamivudine are prescribed. Both patients with NNRTI-associated mutations had been treated with the NNRTI nevirapine, not efavirenz. Rapid emergence of high-level resistance is known to be a potential drawback of nevirapine-including regimens due to the drug's low genetic barrier. No case of dual NRTI-NNRTI was recorded. Mutation T215Y/F, known to confer resistance to zidovudine and stavudine, was also not observed.

Among samples from 27 drug-naïve subjects, none had resistance mutations. These results corroborate findings from regional (Dumans et al. 2002) and national (Brindeiro et al. 1999, 2003) surveillance studies that reported a low prevalence of primary resistance in Brazil. Since antiretroviral drugs are readily available in Miracema since the early 1990s, our results seem to argue against the concern that resource-poor settings should not implement widespread accessibility to standard of care HAART 
combinations due to the possibility of sub-optimal adherence leading to the emergence and spread of drug-resistant strains. Continued surveillance studies on the prevalence of drug resistant strains among treatment naïve and experienced patients will be necessary in large urban centers. However, as the Brazilian epidemic spreads towards the innermost parts of the country, additional investigations will also be needed in these settings.

\section{REFERENCES}

Brindeiro R, Vanderborght B, Caride E, Correa L, Oravec RM, Berro O, Stuyver L, Tanuri A 1999. Sequence diversity of the reverse transcriptase of human immunodeficiency virus type 1 from untreated Brazilian individuals. Antimicrob Agents Chemother 43: 1674-1680.

Brindeiro RM, Diaz RS, Sabino EC, Morgado MG, Pires IL, Brigido L, Dantas MC, Barreira D, Teixeira PR, Tanuri A 2003. Brazilian Network for HIV Drug Resistance Surveillance (HIV-BResNet): a survey of chronically infected individuals. AIDS 17: 1063-1069.

Brito AM, Castilho EA, Szwarcwald CL 2001. AIDS and HIV infection in Brazil: a multifaceted epidemic [in portuguese]. Rev Soc Bras Med Trop 34: 207-217.

Burland TG 2000. DNASTAR's Lasergene sequence analysis software. Methods Mol Biol 132: 71-91.

CDC 1992. 1993 Revised Classification System for HIV infection and expanded surveillance case definition for AIDS among adolescents and adults. MMWR Recomm Rep 41: 961-962.

Cerqueira DM, Amorim RM, Silva RR, Camara GN, Brigido MM, Martins CR 2004. Antiretroviral resistance and genetic diversity of human immunodeficiency virus type 1 isolates from the Federal District, Central Brazil. Mem Inst Oswaldo Cruz 99: 877-882.

Couto-Fernandez JC, Silva-de-Jesus C, Veloso VG, Rachid M, Gracie RS, Chequer-Fernandez SL, Oliveira SM, ArakakiSanchez D, Chequer PJ, Morgado MG 2005. Human immunodeficiency virus type 1 (HIV-1) genotyping in Rio de Janeiro, Brazil: assessing subtype and drug-resistance associated mutations in HIV-1 infected individuals failing highly active antiretroviral therapy. Mem Inst Oswaldo Cruz 100: 73-78.

Dumans AT, Soares MA, Pieniazek D, Kalish ML, De Vroey V, Hertogs K, Tanuri A 2002. Prevalence of protease and reverse transcriptase drug resistance mutations over time in drug-naive human immunodeficiency virus type 1-positive individuals in Rio de Janeiro, Brazil. Antimicrob Agents Chemother 46: 3075-3079.

Eyer-Silva WA, Basílio-de-Oliveira CA, Morgado MG 2005. HIV infection and AIDS in a small municipality in Southeast Brazil. Rev Saude Publica 39: 950-955.

Felsenstein J 1984. Distance methods for inferring phylogenies. Evolution 38: 16-24.

Felsenstein J 1985. Confidence limits on phylogenies: an ap- proach using the bootstrap. Evolution 39: 783-791.

Hecht FM, Grant RM, Petropoulos CJ, Dillon B, Chesney MA, Tian H, Hellmann NS, Bandrapalli NI, Digilio L, Branson B, Kahn JO 1998. Sexual transmission of an HIV1 variant resistant to multiple reverse-transcriptase and protease inhibitors. N Engl J Med 339: 307-311.

IPEA-Instituto de Pesquisas Econômicas e Aplicadas 2003. Atlas do Desenvolvimento Humano no Brasil, Rio de Janeiro.

Kantor R, Machekano R, Gonzales MJ, Dupnik K, Schapiro JM, Shafer RW 2001. Human immunodeficiency virus reverse transcriptase and protease sequence database: an expanded data model integrating natural language text and sequence analysis programs. Nucleic Acids Res 29: 296-299.

Pires IL, Soares MA, Speranza FA, Ishii SK, Vieira MC, Gouvea MI, Guimaraes MA, de Oliveira FE, Magnanini MM, Brindeiro RM, Tanuri A 2004. Prevalence of human immunodeficiency virus drug resistance mutations and subtypes in drug-naive, infected individuals in the army health service of Rio de Janeiro, Brazil. J Clin Microbiol 42: 426-430.

Rodrigues R, Vazquez CM, Colares JK, Custodio RM, Bonasser Filho F, Souza LR, Gianna MC, Marques CC, Brigido LF 2005. Antiretroviral resistance mutations in human immunodeficiency virus type 1 infected patients enrolled in genotype testing at the Central Public Health Laboratory, Sao Paulo, Brazil: preliminary results. Mem Inst Oswaldo Cruz 100: 97-102.

Saitou N, Nei M 1987. The neighbor-joining method: a new method for reconstructing phylogenetic trees. Mol Biol Evol 4: 406-425.

Salminen MO, Carr JK, Burke DS, McCutchan FE 1995. Identification of breakpoints in intergenotypic recombinants of HIV type 1 by bootscanning. AIDS Res Hum Retroviruses 11: 1423-1425.

Siegrist CA, Yerly S, Kaiser L, Wyler CA, Perrin L 1994. Mother to child transmission of zidovudine-resistant HIV-1. Lancet 344: 1771-1772.

Swofford D 1998. PAUP*. Phylogenetic analysis using parsimony (*and other methods). Version 4.0b10. Saunderland, MA, USA: Sinauer Associates, Inc., 1999.

Szwarcwald CL, Bastos FI, Esteves MA, de Andrade CL 2000. The spread of the AIDS epidemic in Brazil from 1987 to 1996: a spatial analysis [in portuguese]. Cad Saúde Pública 16: 7-19.

Tanuri A, Caride E, Dantas MC, Morgado MG, Mello DL, Borges S, Tavares M, Ferreira SB, Santoro-Lopes G, Martins CR, Esteves AL, Diaz RS, Andreo SM, Ferreira LA, Rodrigues R, Reuter T, Cavalcanti AM, de Oliveira SM, de Barbosa HB, Teixeira PR, Chequer PN 2002. Prevalence of mutations related to HIV-1 antiretroviral resistance in Brazilian patients failing HAART. J Clin Virol 25: 39-46.

Thompson JD, Gibson TJ, Plewniak F, Jeanmougin F, Higgins DG 1997. The CLUSTAL_X windows interface: flexible strategies for multiple sequence alignment aided by quality analysis tools. Nucleic Acids Res 25: 4876-4882. 